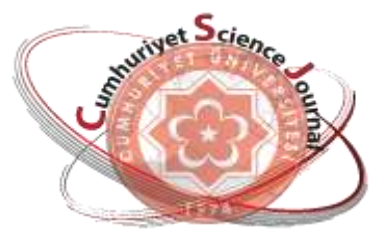

e-ISSN: $2587-246 X$

ISSN: 2587-2680

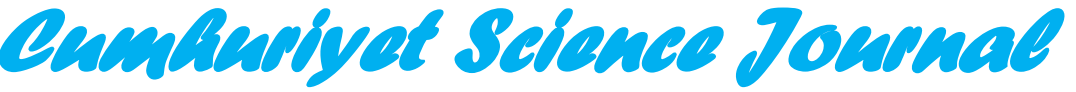

es7

Cumhuriyet Sci. J., Vol.39-1(2018) 71-79

\title{
Some Geometric Properties of The Spacelike Bezier Curve with a Timelike Principal Normal in Minkowski 3-Space
}

\author{
Hatice KUSAK SAMANCI
}

Bitlis Eren Univ. Faculty of Art and Science, Bitlis, TURKEY

Received: 14.10.2017; Accepted: 10.12.2017

http://dx.doi.org/10.17776/csj.344353

\begin{abstract}
The aim of present paper is to introduce and investigate the spacelike Bezier curve with a timelike principal normal in Minkowski 3-space. The Serret-Frenet frame, curvatures and the derivation formulas of the curve at the starting and ending points are studied.
\end{abstract}

Keywords: Bezier curve, causal character, curvatures, Minkowski 3-space

\section{Zamanımsı Asal Normalli Uzayımsı Bezier Eğrisinin Bazı Geometrik Özellikleri}

Özet: Bu çalışmanın amacı Minkowski 3-uzayında zamanımsı asal normalli uzayımsı Bezier eğrisini tantmak ve incelemektir. Eğrinin başlangıç ve bitiş noktasındaki Serret-Frenet çatısı, eğrilikleri ve türev formülleri çalışılmıştır.

Anahtar Kelimeler: Bezier eğrisi, causal karakterler, eğrilikler, Minkowski 3-uzayı

\section{INTRODUCTION}

Minkowski space is founded by German mathematician Hermann Minkowski, [1]. Let $u=\left(u_{1}, u_{2}, u_{3}\right), v=\left(v_{1}, v_{2}, v_{3}\right)$ be vectors in Minkowski 3-space. If the metric $g($,$) is given by$ $g(u, v)=u_{1} v_{1}+u_{2} v_{2}-u_{3} v_{3}$, then the space $R_{1}^{3}=\left(R^{3}, g(),\right)$ is called the Minkowski 3space where the metric $g($,$) is called the$ Lorentzian or Minkowskian metric. Let $\alpha$ be a curve in Minkowski 3 -space and $T$ be the tangent vector for all points of the curve. The curve $\alpha$ is called a spacelike curve if $g(T, T)>0$ or $T=0$, a timelike curve if $g(T, T)<0$ and a lightlike (null) curve if $g(T, T)=0$ and $T \neq 0$ - Furthermore, there are three possibilities depending on the causal character of $T^{\prime}$. If the vector $T^{\prime}$ is timelike, then the equations $N(s)=\frac{T^{\prime}(s)}{\kappa(s)}$ and $B(s)=T(s) \wedge N(s)$ are provided where the $N$ and $B$ are called the principal normal and the binormal vectors

\footnotetext{
* Corresponding author. Email address: hkusak@beu.edu.tr http://dergipark.gov.tr/csj $\quad$ C2016 Faculty of Science, Cumhuriyet University
} 
respectively. The curvature and torsion of $\alpha$ is defined by $\kappa(s)=\left\|T^{\prime}(s)\right\|$ and $\tau=-g\left(N^{\prime}, B\right)$ . Then the Serret- Frenet equations are given with $T^{\prime}=\kappa N, \quad N^{\prime}=\kappa T+\tau B, \quad B^{\prime}=\tau B$ for the spacelike curve with a timelike principal normal, [2]. If the spacelike vectors $u$ and $v$ satisfy the condition $|g(u, v)|<\|u\|_{L}\|v\|_{L}$, then $u \times v$ vector is a timelike vector and the equations $|g(u, v)|=\|u\|_{L}\|v\|_{L} \cos \theta \quad$ and $\|u \times v\|_{\mathrm{L}}=\|u\|_{\mathrm{L}}\|v\|_{\mathrm{L}} \sin \theta$ are used where $\theta$ is the spacelike angle between, $u$ and $v$ spacelike vectors. If the spacelike vectors $u$ and $v$ ensure the condition $|g(u, v)|>\|u\|_{L}\|v\|_{L}$, then $u \times v$ vector is a timelike vector, and the equations $g(u, v)=-\|u\|_{\mathrm{L}}\|v\|_{\mathrm{L}} \cosh \theta \quad$ and $\|u \times v\|_{L}=\|u\|_{L}\|v\|_{L} \sinh \theta$ are satisfied where $\theta$ is the hyperbolic angle between the $u$ and $v$ spacelike vectors. If the vectors $u$ and $v$ are the spacelike vectors provided the equation $|g(u, v)|_{\mathrm{L}}=\|u\|_{\mathrm{L}}\|v\|_{\mathrm{L}}$, then $u \times v$ is a lightlike vector, [1].

On the other hand, the curve $b^{n}(t)=\sum_{i=0}^{n} \mathrm{~b}_{i} B_{i}^{n}(t)$ is called a Bezier curve given with the control points $\mathrm{b}_{0}, \mathrm{~b}_{1}, \ldots, \mathrm{b}_{n}$ for each $t \in[0,1]$ where $B_{i}^{n}(t)=\left(\begin{array}{c}n \\ i\end{array}\right) t^{i}(1-t)^{n-i}$ are called Bernstein polynomials. Bezier curves were first developed by a French engineer (1958-1960), Pierre Bezier (1910-1999) and a French mathematician Paul de Faget de Casteljau (1930) independently with different mathematical approaches. Bezier curves provide easiness in design processes because they are controllable in such a way that Bezier curves can be used almost in every design of any of the products used now. The Bezier curves have a wide variety of usage in design because of being interactive, in production because of the easiness in usage $[3,4,5]$. Spacelike Bezier curves in the three dimensional Minkowski 3-space was firstly introduced by G.H. Georgiev in 2008. The curvature and torsion of the spacelike Bezier curves at the beginning point, i.e $\kappa(0)$ and $\tau(0)$ were given in the paper [6]. In the $[7,8]$ the authors considered a spacelike quadratic and cubic Bezier curves in Minkowski 2-plane $\mathrm{R}_{1}^{2}$. Furthermore, similarly a spacelike curve with a spacelike principle normal in Minkowski 3-space were studied in [9].

Our main intention of this paper to investigate some differential geometric properties of the spacelike Bezier curve with a timelike principal normal, e.g. curvature, torsion, Serret-Frenet equations and the derivativation formula of Serret-Frenet equations.

\section{MAIN RESULTS}

Let $b_{0}, b_{1}, \ldots, b_{n} \in \mathrm{R}_{1}^{3}$ be the control points, and $b^{n}(t)$ be a spacelike Bezier curve, which does not have unit speed. If the Bezier curve $b^{n}(t)$ is spacelike curve, then the tangent $T$ must be spacelike vector. However, in case $T^{\prime}$ i.e. the principle normal is timelike $b^{n}(t)$ spacelike Bezier curve is called as "the spacelike Bézier curve with timelike principle normal". The orthonormal frame $\left.\{T, N, B\}\right|_{\mathrm{t}=0}$ of the $\mathrm{b}^{\mathrm{n}}(\mathrm{t})$ spacelike Bezier curve in the $t=0$ starting point is include the $T$ spacelike vector, $N$ timelike vector and $B$ spacelike vector. So the conditions $g(T, T)=1, g(N, N)=-1, g(B, B)=1$, $g(T, N)=0, g(T, B)=0, g(N, B)=0 \quad$ are satisfied. If the Bezier curve $b^{n}(t)$ is spacelike, then $g\left(\frac{d b^{n}(t)}{d t}, \frac{d b^{n}(t)}{d t}\right)>0$. Therefore, the 
length of the speed vector and the arc parameter

of

$$
b^{n}(t)
$$

are

$v=\left\|\frac{d b^{n}(t)}{d t}\right\|_{\mathrm{L}}=\sqrt{g\left(\frac{d b^{n}(t)}{d t}, \frac{d b^{n}(t)}{d t}\right)}$ and

$s=\int_{t_{0}}^{t_{1}} \sqrt{g\left(\frac{d b^{n}(t)}{d t}, \frac{d b^{n}(t)}{d t}\right)} d t$, recpectively. If

the orthonormal frame vectors are $T$ spacelike, $N$ timelike, $B$ spacelike, then the vectoral products are satisfy the equations $T \wedge_{\mathrm{L}} N=-B$, $N \wedge_{\mathrm{L}} B=-T$, and $B \wedge_{\mathrm{L}} T=N$.

Let $b_{0}, b_{1}, \ldots, b_{n} \in \mathrm{R}_{1}^{3}$ be the control points of the spacelike Bezier curve with timelike principal normal in Minkowski 3-space. The convex polygon vectors $\Delta b_{i}$ are spacelike vectors which are existed in the same spacelike cone. If the vectors $\Delta b_{0}$ and $\Delta b_{1}$ are spacelike, the vectors have three conditions for using inner and exterior product as following:

Condition (1). $\left|g\left(\Delta b_{0}, \Delta b_{1}\right)\right|<\left\|\Delta b_{0}\right\|_{L} \cdot\left\|\Delta b_{1}\right\|_{L}$

Condition (2). $\left|g\left(\Delta b_{0}, \Delta b_{1}\right)\right|>\left\|\Delta b_{0}\right\|_{L} \cdot\left\|\Delta b_{1}\right\|_{L}$

Condition (3). $\left|g\left(\Delta b_{0}, \Delta b_{1}\right)\right|=\left\|\Delta b_{0}\right\|_{\mathrm{L}} \cdot\left\|\Delta b_{1}\right\|_{\mathrm{L}}$

In our paper, we will deal with Condition (1) and (2).

Theorem 2.1. If $\Delta b_{0}$ and $\Delta b_{1}$ vectors satisfy the Condition (1), then the Serret-Frenet frame $\left.\{T, N, B\}\right|_{t=0}$ in the starting point $t=0$ is obtained by $\left.T\right|_{t=0}=\frac{\Delta b_{0}}{\sqrt{g\left(\Delta b_{0}, \Delta b_{0}\right)}}$ $\left.N\right|_{t=0}=\frac{\Delta b_{0}}{\left\|\Delta b_{0}\right\|_{\mathrm{L}}} \cot \theta-\frac{\Delta b_{1}}{\left\|\Delta b_{1}\right\|_{\mathrm{L}}} \operatorname{cosec} \theta$

$$
\left.B\right|_{t=0}=\frac{\Delta b_{0} \wedge_{\mathrm{L}} \Delta b_{1}}{\left\|\Delta b_{0}\right\|_{\mathrm{L}} \cdot\left\|\Delta b_{1}\right\|_{\mathrm{L}} \cdot \sin \theta} .
$$

Proof: Since $b^{n}(t)$ is a spacelike Bezier curve, the tangent vector must also be spacelike. For this reason $\left\|\Delta b_{0}\right\|_{L}=\sqrt{g\left(\Delta b_{0}, \Delta b_{0}\right)}$. The tangent vector in the starting point is given with the following formula:

$$
\left.T\right|_{t=0}=\frac{\frac{d b^{n}(t)}{d t}}{\left\|\frac{d b^{n}(t)}{d t}\right\|_{L}}=\frac{\Delta b_{0}}{\sqrt{g\left(\Delta b_{0}, \Delta b_{0}\right)}} .
$$

Then the binormal vector is calculated by

$$
\begin{aligned}
& \left.B\right|_{t=0}=\left.\frac{\frac{d b^{n}(t)}{d t} \wedge_{\mathrm{L}} \frac{d^{2} b^{n}(t)}{d t^{2}}}{\left\|\frac{d b^{n}(t)}{d t} \wedge_{\mathrm{L}} \frac{d^{2} b^{n}(t)}{d t^{2}}\right\|_{\mathrm{L}}}\right|_{t=0} \\
& =\frac{n \cdot \Delta b_{0} \wedge_{\mathrm{L}}\left[n \cdot(n-1)\left\{\Delta b_{1}-\Delta b_{0}\right\}\right]}{\left\|n \cdot \Delta b_{0} \wedge_{\mathrm{L}}\left[n \cdot(n-1)\left\{\Delta b_{1}-\Delta b_{0}\right\}\right]\right\|_{\mathrm{L}}} \\
& =\frac{\Delta b_{0} \wedge_{\mathrm{L}} \Delta b_{1}-\Delta b_{0} \wedge_{\mathrm{L}} \Delta b_{0}}{\left\|\Delta b_{0} \wedge_{\mathrm{L}} \Delta b_{1}-\Delta b_{0} \wedge_{\mathrm{L}} \Delta b_{0}\right\|_{\mathrm{L}}} \\
& =\frac{\Delta b_{0} \wedge_{\mathrm{L}} \Delta b_{1}}{\left\|\Delta b_{0}\right\|_{\mathrm{L}}\left\|\Delta b_{1}\right\|_{\mathrm{L}} \sin \theta} .
\end{aligned}
$$

Since $T$ spacelike, $N$ timelike and $B$ spacelike, the principal normal vector $N$ is provided by

$$
\begin{aligned}
& \left.N\right|_{t=0}=\left.\left.B\right|_{t=0} \wedge_{\mathrm{L}} T\right|_{t=0} \\
& =\frac{\Delta b_{0} \wedge_{\mathrm{L}} \Delta b_{1}}{\left\|\Delta b_{0} \wedge_{\mathrm{L}} \Delta b_{1}\right\|_{\mathrm{L}}} \wedge_{\mathrm{L}} \frac{\Delta b_{0}}{\left\|\Delta b_{0}\right\|_{\mathrm{L}}} \\
& =\left(\frac{-g\left(\Delta b_{0}, \Delta b_{0}\right) \cdot \Delta b_{1}+g\left(\Delta b_{1}, \Delta b_{0}\right) \cdot \Delta b_{0}}{\left\|\Delta b_{0}\right\|_{\mathrm{L}} \cdot\left\|\Delta b_{1}\right\|_{\mathrm{L}} \sin \theta \cdot\left\|\Delta b_{0}\right\|_{\mathrm{L}}}\right)
\end{aligned}
$$


$=\frac{\Delta b_{0}}{\left\|\Delta b_{0}\right\|_{L}} \cot \theta-\frac{\Delta b_{1}}{\left\|\Delta b_{1}\right\|_{L}} \operatorname{cosec} \theta$

where $\theta=\left(\Delta b_{0}, \Delta b_{1}\right)$ is the spacelike angle between the $\Delta b_{0}$ and $\Delta b_{1}$ spacelike vector.

Theorem 2.2. If $\Delta b_{0}$ and $\Delta b_{1}$ vectors satisfy the Condition (2), then the Serret-Frenet frame $\left.\{T, N, B\}\right|_{t=0}$ in the starting point $t=0$ is provided by

$$
\begin{aligned}
\left.T\right|_{t=0} & =\frac{\Delta b_{0}}{\sqrt{g\left(\Delta b_{0}, \Delta b_{0}\right)}} \\
\left.N\right|_{t=0} & =-\frac{\Delta b_{0}}{\left\|\Delta b_{0}\right\|_{\mathrm{L}}} \operatorname{coth} \varphi-\frac{\Delta b_{1}}{\left\|\Delta b_{1}\right\|_{\mathrm{L}}} \operatorname{csch} \varphi \\
\left.B\right|_{t=0} & =\frac{\Delta b_{0} \wedge_{\mathrm{L}} \Delta b_{1}}{\left\|\Delta b_{0}\right\|_{\mathrm{L}} \cdot\left\|\Delta b_{1}\right\|_{\mathrm{L}} \cdot \sinh \varphi} .
\end{aligned}
$$

Proof: The proof of the tangent vector $\left.T\right|_{t=0}$ is the same as the Theo.2.1. The binormal vector $\left.B\right|_{t=0}$ is found by

$$
\begin{aligned}
& \left.B\right|_{t=0}=\frac{\frac{d b^{n}(t)}{d t} \wedge_{\mathrm{L}} \frac{d^{2} b^{n}(t)}{d t^{2}}}{\left.\left\|\frac{d b^{n}(t)}{d t} \wedge_{\mathrm{L}} \frac{d^{2} b^{n}(t)}{d t^{2}}\right\|_{\mathrm{L}}\right|_{t=0}} \\
& =\frac{n \cdot \Delta b_{0} \wedge_{\mathrm{L}}\left[n \cdot(n-1)\left\{\Delta b_{1}-\Delta b_{0}\right\}\right]}{\left\|n \cdot \Delta b_{0} \wedge_{\mathrm{L}}\left[n \cdot(n-1)\left\{\Delta b_{1}-\Delta b_{0}\right\}\right]\right\|_{\mathrm{L}}} \\
& =\frac{\Delta b_{0} \wedge_{\mathrm{L}} \Delta b_{1}}{\left\|\Delta b_{0} \wedge_{\mathrm{L}} \Delta b_{1}\right\|_{\mathrm{L}}}=\frac{\Delta b_{0} \wedge_{\mathrm{L}} \Delta b_{1}}{\left\|\Delta b_{0}\right\|_{\mathrm{L}}\left\|\Delta b_{1}\right\|_{\mathrm{L}} \sinh \varphi}
\end{aligned}
$$

where $\varphi=\left(\Delta b_{0}, \Delta b_{1}\right)$ is called a hiperbolic angle. Since $T$ spacelike, $N$ timelike, $B$ spacelike, the equation of the principal normal $N$ will be taken by $N=B \wedge_{\mathrm{L}} T$. Since $\Delta b_{0}$ is spacelike, the norm is given by $\left\|\Delta b_{0}\right\|^{2}=g\left(\Delta b_{0}, \Delta b_{0}\right)$. Thus the principal normal is

$$
\begin{aligned}
& \left.N\right|_{t=0}=\left.\left.B\right|_{t=0} \wedge_{\mathrm{L}} T\right|_{t=0} \\
& =\frac{\Delta b_{0} \wedge_{\mathrm{L}} \Delta b_{1}}{\left\|\Delta b_{0} \wedge_{\mathrm{L}} \Delta b_{1}\right\|_{\mathrm{L}}} \wedge_{\mathrm{L}} \frac{\Delta b_{0}}{\left\|\Delta b_{0}\right\|_{\mathrm{L}}} \\
& =\left(\frac{-g\left(\Delta b_{0}, \Delta b_{0}\right) \cdot \Delta b_{1}+g\left(\Delta b_{1}, \Delta b_{0}\right) \cdot \Delta b_{0}}{\left\|\Delta b_{0}\right\|_{\mathrm{L}} \cdot\left\|\Delta b_{1}\right\|_{\mathrm{L}} \sinh \varphi \cdot\left\|\Delta b_{0}\right\|_{\mathrm{L}}}\right) \\
& =-\frac{\Delta b_{0}}{\left\|\Delta b_{0}\right\|_{\mathrm{L}}} \operatorname{coth} \theta-\frac{\Delta b_{1}}{\left\|\Delta b_{1}\right\|_{\mathrm{L}}} \operatorname{csch} \theta .
\end{aligned}
$$

Theorem 2.3. Let $b_{0}, b_{1}, \ldots, b_{n}$ be the spacelike control points. If the Condition (1) is satisfied, then the curvature and torsion of the spacelike Bezier curve $b^{n}(t)$ with timelike principal normal at the starting point $t=0$ are

$\left.\kappa\right|_{t=0}=\frac{n-1}{n} \frac{\left\|\Delta b_{1}\right\|}{\left\|\Delta b_{0}\right\|^{2}} \cdot \sin \theta$

$$
\left.\tau\right|_{t=0}=-\frac{n-2}{n} \frac{\operatorname{det}\left(\Delta b_{0}, \Delta b_{1}, \Delta b_{2}\right)}{\left\|\Delta b_{0} \wedge_{\mathrm{L}} \Delta b_{1}\right\|^{2}} .
$$

Proof: $\Delta b_{0}$ and $\Delta b_{1}$ vectors that ensure the Condition (1), the curvature at the starting point for spacelike vectors is calculated with:

$$
\begin{aligned}
\left.\kappa\right|_{t=0} & =\left.\frac{\left\|\frac{d b^{n}(t)}{d t} \wedge_{\mathrm{L}} \frac{d^{2} b^{n}(t)}{d t^{2}}\right\|_{\mathrm{L}}}{\left\|\frac{d b^{n}(t)}{d t}\right\|_{\mathrm{L}}^{3}}\right|_{t=0} \\
& =\frac{n-1}{n} \frac{\left\|\Delta b_{0} \wedge_{\mathrm{L}}\left(\Delta b_{1}-\Delta b_{0}\right)\right\|_{\mathrm{L}}}{\left\|\Delta b_{0}\right\|_{\mathrm{L}}^{3}}
\end{aligned}
$$

$$
=\frac{n-1}{n} \frac{\left\|\Delta b_{0} \wedge_{\mathrm{L}} \Delta b_{1}\right\|_{\mathrm{L}}}{\left\|\Delta b_{0}\right\|_{\mathrm{L}}^{3}}
$$




$$
=\frac{n-1}{n} \frac{\left\|\Delta b_{1}\right\|_{\mathrm{L}}}{\left\|\Delta b_{0}\right\|_{\mathrm{L}}^{2}} \cdot \sin \theta
$$

Now, let us find the torsion at the starting point.

$$
\begin{aligned}
& \left.\tau\right|_{t=0}=\left.\frac{g\left(\frac{d b^{n}(t)}{d t} \wedge_{\mathrm{L}} \frac{d^{2} b^{n}(t)}{d t^{2}}, \frac{d^{3} b^{n}(t)}{d t^{3}}\right)}{\left\|\frac{d b^{n}(t)}{d t} \wedge_{\mathrm{L}} \frac{d^{2} b^{n}(t)}{d t^{2}}\right\|_{\mathrm{L}}^{2}}\right|_{t=0} \\
& =\frac{n-2}{n} \frac{g\left(\Delta b_{0} \wedge_{I L} \Delta b_{1}, \Delta b_{2}-2 \Delta b_{1}+\Delta b_{0}\right)}{\left\|\Delta b_{0} \wedge_{\mathrm{L}}\left(\Delta b_{1}-\Delta b_{0}\right)\right\|_{\mathrm{L}}^{2}} \\
& =\frac{n-2}{n} \frac{g\left(\Delta b_{0} \wedge_{\mathrm{L}} \Delta b_{1}, \Delta b_{2}\right)}{\left\|\Delta b_{0} \wedge_{\mathrm{L}}\left(\Delta b_{1}-\Delta b_{0}\right)\right\|_{\mathrm{L}}^{2}} \\
& =-\frac{n-2}{n} \frac{\operatorname{det}\left(\Delta b_{0}, \Delta b_{1}, \Delta b_{2}\right)}{\left\|\Delta b_{0} \wedge_{\mathrm{L}} \Delta b_{1}\right\|_{\mathrm{L}}^{2}}
\end{aligned}
$$

Theorem 2.4. Let $b_{0}, b_{1}, \ldots, b_{n}$ be the spacelike control points. If $\Delta b_{0}$ and $\Delta b_{1}$ vectors satify the Condition (2), the curvature and torsion of the spacelike Bezier curve with timelike principal normal at the starting point are

$$
\begin{aligned}
& \left.\kappa\right|_{t=0}=\frac{n-1}{n} \frac{\left\|\Delta b_{1}\right\|_{\mathrm{L}}}{\left\|\Delta b_{0}\right\|_{\mathrm{L}}^{2}} \cdot \sinh \varphi \\
& \left.\tau\right|_{t=0}=-\frac{n-2}{n} \frac{\operatorname{det}\left(\Delta b_{0}, \Delta b_{1}, \Delta b_{2}\right)}{\left\|\Delta b_{0} \wedge_{\mathrm{L}} \Delta b_{1}\right\|_{\mathrm{L}}^{2}} .
\end{aligned}
$$

Proof: The curvature at the starting point is

$$
\left.\kappa\right|_{t=0}=\left.\frac{\left\|\frac{d b^{n}(t)}{d t} \wedge_{\mathrm{L}} \frac{d^{2} b^{n}(t)}{d t^{2}}\right\|_{\mathrm{L}}}{\left\|\frac{d b^{n}(t)}{d t}\right\|_{\mathrm{L}}^{3}}\right|_{t=0}
$$

$$
=\frac{n-1}{n} \frac{\left\|\Delta b_{0} \wedge_{\mathrm{L}}\left(\Delta b_{1}-\Delta b_{0}\right)\right\|_{\mathrm{L}}}{\left\|\Delta b_{0}\right\|_{\mathrm{L}}^{3}}
$$

$$
=\frac{n-1}{n} \frac{\left\|\Delta b_{1}\right\|_{\mathrm{L}}}{\left\|\Delta b_{0}\right\|_{\mathrm{L}}^{2}} \cdot \sinh \varphi .
$$

Here, the torsion equation for the Condition (2) can be proven similar method with Theo.2.3.

Theorem 2.5. For the spacelike vectors provided the Condition (1), the Serret-Frenet frame derivation formula of the at the $t=0$ of the curve $b^{n}(t)$ is

$$
\begin{aligned}
T^{\prime}= & (n-1) \frac{\left\|\Delta b_{1}\right\|_{\mathrm{L}}}{\left\|\Delta b_{0}\right\|_{\mathrm{L}}} \cdot \sin \theta \cdot N \\
N^{\prime}= & (n-1) \frac{\left\|\Delta b_{1}\right\|_{\mathrm{L}}}{\left\|\Delta b_{0}\right\|_{\mathrm{L}}} \cdot \sin \theta \cdot T \\
& -(n-2)\left\|\Delta b_{0}\right\|_{\mathrm{L}} \frac{\operatorname{det}\left(\Delta b_{0}, \Delta b_{1}, \Delta b_{2}\right)}{\left\|\Delta b_{0} \wedge_{\mathrm{L}} \Delta b_{1}\right\|_{\mathrm{L}}^{2}} \cdot B \\
B^{\prime}= & -(n-2)\left\|\Delta b_{0}\right\|_{\mathrm{L}} \frac{\operatorname{det}\left(\Delta b_{0}, \Delta b_{1}, \Delta b_{2}\right)}{\left\|\Delta b_{0} \wedge_{\mathrm{L}} \Delta b_{1}\right\|_{\mathrm{L}}^{2}} \cdot N
\end{aligned}
$$

Proof: The Frenet derivation formula for spacelike curve with timelike principal normal is

$$
\left(\begin{array}{l}
T^{\prime} \\
N^{\prime} \\
B^{\prime}
\end{array}\right)=\left(\begin{array}{ccc}
0 & \kappa v_{1} & 0 \\
\kappa v_{1} & 0 & \tau v_{1} \\
0 & \tau v_{1} & 0
\end{array}\right)\left(\begin{array}{l}
T \\
N \\
B
\end{array}\right)
$$

where $v_{1}=n\left\|b_{1}-b_{0}\right\|=n\left\|\Delta b_{0}\right\|$. Hence we get

$$
\begin{aligned}
T^{\prime} & =\kappa v_{1} \cdot N \\
& =\frac{(n-1)}{n} \frac{\left\|\Delta b_{1}\right\|_{\mathrm{L}}}{\left\|\Delta b_{0}\right\|_{\mathrm{L}}^{2}} \sin \theta \cdot n\left\|\Delta b_{0}\right\|_{\mathrm{L}} \cdot N \\
& =(n-1) \frac{\left\|\Delta b_{1}\right\|_{\mathrm{L}}}{\left\|\Delta b_{0}\right\|_{\mathrm{L}}} \sin \theta \cdot N
\end{aligned}
$$




$$
\begin{aligned}
N^{\prime}= & \kappa v_{1} T+\tau v_{1} B \\
= & (n-1) \frac{\left\|\Delta b_{1}\right\|_{\mathrm{L}}}{\left\|\Delta b_{0}\right\|_{\mathrm{L}}} \sin \theta \cdot T \\
& -(n-2)\left\|\Delta b_{0}\right\|_{\mathrm{L}} \frac{\operatorname{det}\left(\Delta b_{0}, \Delta b_{1}, \Delta b_{2}\right)}{\left\|\Delta b_{0} \wedge_{\mathrm{L}} \Delta b_{1}\right\|_{\mathrm{L}}^{2}} \cdot B \\
\left.B^{\prime}\right|_{t=0}=\tau v_{1} N & \\
=- & (n-2)\left\|\Delta b_{0}\right\|_{\mathrm{L}} \frac{\operatorname{det}\left(\Delta b_{0}, \Delta b_{1}, \Delta b_{2}\right)}{\left\|\Delta b_{0} \wedge_{\mathrm{L}} \Delta b_{1}\right\|_{\mathrm{L}}^{2}} \cdot N
\end{aligned}
$$

Theorem 2.6. For the spacelike vectors $\Delta b_{0}$ and $\Delta b_{1}$ vectors that satisfy the Condition (2), the derivation formula of the Serret-Frenet frame is yield by

$$
\begin{aligned}
T^{\prime} & =(n-1) \frac{\left\|\Delta b_{1}\right\|_{\mathrm{L}}}{\left\|\Delta b_{0}\right\|_{\mathrm{L}}} \cdot \sinh \varphi \cdot N \\
N^{\prime} & =(n-1) \frac{\left\|\Delta b_{1}\right\|_{\mathrm{L}}}{\left\|\Delta b_{0}\right\|_{\mathrm{L}}} \cdot \sinh \varphi \cdot T \\
& -(n-2)\left\|\Delta b_{0}\right\|_{\mathrm{L}} \frac{\operatorname{det}\left(\Delta b_{0}, \Delta b_{1}, \Delta b_{2}\right)}{\left\|\Delta b_{0} \wedge_{\mathrm{L}} \Delta b_{1}\right\|_{\mathrm{L}}^{2}} \cdot B \\
B^{\prime} & =-(n-2)\left\|\Delta b_{0}\right\|_{\mathrm{L}} \frac{\operatorname{det}\left(\Delta b_{0}, \Delta b_{1}, \Delta b_{2}\right)}{\left\|\Delta b_{0} \wedge_{\mathrm{L}} \Delta b_{1}\right\|_{\mathrm{L}}^{2}} \cdot N .
\end{aligned}
$$

Proof: Following calculations give us the derivation formula of Serret-Frenet frame:

$$
\begin{aligned}
\left.T^{\prime}\right|_{t=0} & =\kappa v_{1} \cdot N \\
& =\frac{n-1}{n} \frac{\left\|\Delta b_{1}\right\|_{\mathrm{L}}}{\left\|\Delta b_{0}\right\|_{\mathrm{L}}^{2}} \cdot \sinh \varphi \cdot n\left\|\Delta b_{0}\right\|_{\mathrm{L}} N \\
& =(n-1) \frac{\left\|\Delta b_{1}\right\|_{\mathrm{L}}}{\left\|\Delta b_{0}\right\|_{\mathrm{L}}} \cdot \sinh \varphi \cdot N
\end{aligned}
$$

$$
\begin{aligned}
\left.N^{\prime}\right|_{t=0}=\kappa v_{1} T+\tau v_{1} B & \frac{(n-1)}{n} \frac{\left\|\Delta b_{1}\right\|_{\mathrm{L}}}{\left\|\Delta b_{0}\right\|_{\mathrm{L}}^{2}} \cdot \sinh \varphi \cdot n\left\|\Delta b_{0}\right\|_{\mathrm{L}} T \\
& -\frac{n-2}{n} \frac{\operatorname{det}\left(\Delta b_{0}, \Delta b_{1}, \Delta b_{2}\right)}{\left\|\Delta b_{0} \wedge_{\mathrm{L}} \Delta b_{1}\right\|_{\mathrm{L}}^{2}} n\left\|\Delta b_{0}\right\|_{\mathrm{L}} \cdot B \\
= & (n-1) \frac{\left\|\Delta b_{1}\right\|_{\mathrm{L}}}{\left\|\Delta b_{0}\right\|_{\mathrm{L}}} \cdot \sinh \varphi \cdot T \\
& -(n-2)\left\|\Delta b_{0}\right\|_{\mathrm{L}} \frac{\operatorname{det}\left(\Delta b_{0}, \Delta b_{1}, \Delta b_{2}\right)}{\left\|\Delta b_{0} \wedge \Delta b_{1}\right\|_{\mathrm{L}}^{2}} \cdot B \\
\left.B^{\prime}\right|_{t=0}=\tau v_{1} N & -\frac{(n-2)}{n} \frac{\left(\Delta b_{0}, \Delta b_{1}, \Delta b_{2}\right)}{\left\|\Delta b_{0} \wedge \wedge_{\mathrm{L}} \Delta b_{1}\right\|_{\mathrm{L}}^{2}} \cdot n \|_{\Delta b_{0} \|_{\mathrm{L}}} \cdot N \\
= & -(n-2)\left\|\Delta b_{0}\right\|_{\mathrm{L}} \frac{\operatorname{det}\left(\Delta b_{0}, \Delta b_{1}, \Delta b_{2}\right)}{\left\|\Delta b_{0} \wedge \Delta b_{1}\right\|_{\mathrm{L}}^{2}} \cdot N . \\
= &
\end{aligned}
$$

Let $b_{i} \in \mathrm{R}_{1}^{3}$ be the control points of the spacelike Bezier curve with principal normal.

Theorem 2.7. If the $\Delta b_{n-1}$ and $\Delta b_{n-2}$ vectors are satisfy Condition (1), the Serret-Frenet frame $\left.\{T, N, B\}\right|_{t=1}$ at the ending point $t=1$ is obtained by

$$
\begin{aligned}
& \left.T\right|_{t=1}=\frac{\Delta b_{n-1}}{\sqrt{g\left(\Delta b_{n-1}, \Delta b_{n-1}\right)}} \\
& \left.N\right|_{t=1}=\frac{\Delta b_{n-2}}{\left\|\Delta b_{n-2}\right\|_{\mathrm{L}}} \operatorname{cosec} \theta-\frac{\Delta b_{n-1}}{\left\|\Delta b_{n-1}\right\|_{\mathrm{L}}} \cot \theta \\
& \left.B\right|_{t=1}=-\frac{\Delta b_{n-1} \wedge_{\mathrm{L}} \Delta b_{n-2}}{\left\|\Delta b_{n-1}\right\|_{\mathrm{L}} \cdot\left\|\Delta b_{n-2}\right\|_{\mathrm{L}} \sin \theta}
\end{aligned}
$$

Proof: The proof is similar with Theo. 2.1. 
Theorem 2.8. If the $\Delta b_{n-1}$ and $\Delta b_{n-2}$ vectors satisfy Condition (2), the Serret-Frenet frame $\left.\{T, N, B\}\right|_{t=1}$ at the ending point $t=1$ is obtained by

$$
\begin{aligned}
& \left.T\right|_{t=1}=\frac{\Delta b_{n-1}}{\sqrt{g\left(\Delta b_{n-1}, \Delta b_{n-1}\right)}} \\
& \left.N\right|_{t=1}=\frac{\Delta b_{n-2}}{\left\|\Delta b_{n-2}\right\|_{\mathrm{L}}} \operatorname{csch} \varphi+\frac{\Delta b_{n-1}}{\left\|\Delta b_{n-1}\right\|_{\mathrm{L}}} \operatorname{coth} \varphi \\
& \left.B\right|_{t=1}=-\frac{\Delta b_{n-1} \wedge_{\mathrm{L}} \Delta b_{n-2}}{\left\|\Delta b_{n-1}\right\|_{\mathrm{L}} \cdot\left\|\Delta b_{n-2}\right\|_{\mathrm{L}} \sinh \varphi} .
\end{aligned}
$$

Proof: The proof is similar with Theo. 2.2.

Theorem 2.9. For the spacelike vectors that provide the Condition (1), the curvature and torsion of the curve at the ending point are obtained by

$$
\begin{aligned}
& \left.\kappa\right|_{t=1}=\frac{n-1}{n} \frac{\left\|\Delta b_{n-2}\right\|_{\mathrm{L}}}{\left\|\Delta b_{n-1}\right\|_{\mathrm{L}}^{2}} \cdot \sin \theta \\
& \left.\tau\right|_{t=1}=\frac{n-2}{n} \frac{\operatorname{det}\left(\Delta b_{n-1}, \Delta b_{n-2}, \Delta b_{n-3}\right)}{\left\|\Delta b_{n-1} \wedge_{\mathrm{L}} \Delta b_{n-2}\right\|_{\mathrm{L}}^{2}}
\end{aligned}
$$

Proof: The proof is similar with Theo. 2.3.

Theorem 2.10. If the spacelike vectors satisfy the Condition (2), the curvature and torsion are given by

$$
\begin{aligned}
& \left.\kappa\right|_{t=1}=\frac{n-1}{n} \frac{\left\|\Delta b_{n-2}\right\|_{\mathrm{L}}}{\left\|\Delta b_{n-1}\right\|_{\mathrm{L}}^{2}} \cdot \sinh \varphi \\
& \left.\tau\right|_{t=1}=\frac{n-2}{n} \frac{\operatorname{det}\left(\Delta b_{n-1}, \Delta b_{n-2}, \Delta b_{n-3}\right)}{\left\|\Delta b_{n-1} \wedge_{\mathrm{L}} \Delta b_{n-2}\right\|_{\mathrm{L}}^{2}} .
\end{aligned}
$$

Proof: The proof is similar with Theo. 2.4.

Theorem 2.11. If the spacelike vectors $\Delta b_{n-1}$ and $\Delta b_{n-2}$ vectors that ensure the Condition (1), then the derivation formula of Serret-Frenet frame at the ending point $t=1$ is calculated by

$$
\begin{aligned}
& T^{\prime}=(n-1) \frac{\left\|\Delta b_{n-2}\right\|_{\mathrm{L}}}{\left\|\Delta b_{n-1}\right\|_{\mathrm{L}}} \sin \theta \cdot N \\
& N^{\prime}=(n-1) \frac{\left\|\Delta b_{n-2}\right\|_{\mathrm{L}}}{\left\|\Delta b_{n-1}\right\|_{\mathrm{L}}} \sin \theta \cdot T \\
& +(n-2)\left\|\Delta b_{n-1}\right\|_{\mathrm{L}} \frac{\operatorname{det}\left(\Delta b_{n-1}, \Delta b_{n-2}, \Delta b_{n-3}\right)}{\left\|\Delta b_{n-1} \wedge_{\mathrm{L}} \Delta b_{n-2}\right\|_{\mathrm{L}}^{2}} \cdot B \\
& B^{\prime}=(n-2) \frac{\operatorname{det}\left(\Delta b_{n-1}, \Delta b_{n-2}, \Delta b_{n-3}\right)}{\left\|\Delta b_{n-1} \wedge \Delta b_{n-2}\right\|_{\mathrm{L}}^{2}}\left\|\Delta b_{n-1}\right\|_{\mathrm{L}} . N .
\end{aligned}
$$

Proof: The proof is similar with Theo. 2.5.

Theorem 2.12. If the spacelike vectors $\Delta b_{n-1}$ and $\Delta b_{n-2}$ vectors that ensure the Condition (2), then the derivation formula of Serret-Frenet frame at the ending point $t=1$ is calculated by

$$
\begin{aligned}
T^{\prime}= & (n-1) \frac{\left\|\Delta b_{n-2}\right\|_{\mathrm{L}}}{\left\|\Delta b_{n-1}\right\|_{\mathrm{L}}} \sinh \varphi \cdot N \\
N^{\prime}= & (n-1) \frac{\left\|\Delta b_{n-2}\right\|_{\mathrm{L}}}{\left\|\Delta b_{n-1}\right\|_{\mathrm{L}}} \sinh \varphi \cdot T \\
& +(n-2)\left\|\Delta b_{n-1}\right\|_{\mathrm{L}} \frac{\operatorname{det}\left(\Delta b_{n-1}, \Delta b_{n-2}, \Delta b_{n-3}\right)}{\left\|\Delta b_{n-1} \wedge_{\mathrm{L}} \Delta b_{n-2}\right\|_{\mathrm{L}}^{2}} \cdot B \\
B^{\prime}= & (n-2) \frac{\operatorname{det}\left(\Delta b_{n-1}, \Delta b_{n-2}, \Delta b_{n-3}\right)}{\left\|\Delta b_{n-1} \wedge \Delta b_{n-2}\right\|_{\mathrm{L}}^{2}}\left\|\Delta b_{n-1}\right\|_{\mathrm{L}} \cdot N .
\end{aligned}
$$

Proof: The proof is similar with Theo.2.6.

\section{NUMERIC EXAMPLE}

Consider a cubic Bezier curve $b^{n}(t)$ with spacelike control points $b_{0}=(3,3,2)$, $b_{1}=(4,4,1), b_{2}=(5,7,3), \quad b_{3}=(6,3,5)$ in Minkowski 3-space. 


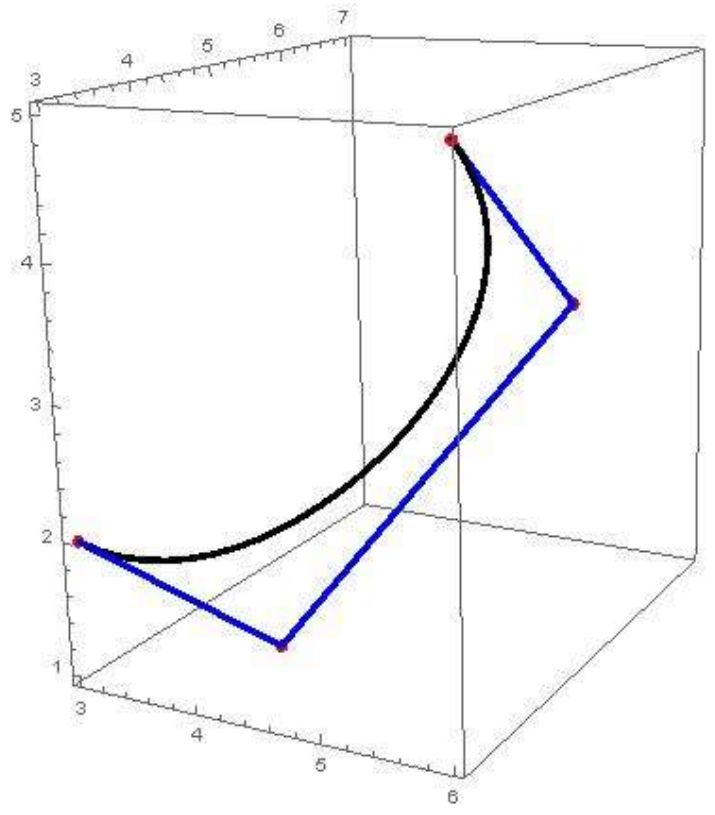

Figure 1. The cubic Bezier curve.

Then the spacelike convex hull is found by the vectors $\quad \Delta b_{0}=(1,1,-1), \quad \Delta b_{1}=(1,3,2)$, $\Delta b_{2}=(1,-4,2)$. The first, second and third derivations at $t=0$ are

$\left.\frac{d b^{n}(t)}{d t}\right|_{t=0}=(-3,3,-3)$

$\left.\frac{d^{2} b^{n}(t)}{d t^{2}}\right|_{t=0}=(0,12,18)$

$\left.\frac{d^{3} b^{n}(t)}{d t^{3}}\right|_{t=0}=(0,-54,-18)$.

The norms $\left\|\Delta b_{0}\right\|_{L}=1$ and $\left\|\Delta b_{1}\right\|_{L}=\sqrt{14}$ are calculated. Because of the equation $\left|g\left(\Delta b_{0}, \Delta b_{1}\right)\right|=6, \quad$ the inequality $\left|g\left(\Delta b_{0}, \Delta b_{1}\right)\right|>\left\|\Delta b_{0}\right\|_{\mathrm{L}} \cdot\left\|\Delta b_{1}\right\|_{\mathrm{L}} \quad$ is $\quad$ satisfied. Hence the equations
$g\left(\Delta b_{0}, \Delta b_{1}\right)=-\left\|\Delta b_{0}\right\|_{L} \cdot\left\|\Delta b_{1}\right\|_{L} \cosh \theta$,

$\|u \times v\|_{L}=\|u\|_{L}\|v\|_{L} \sinh \theta$

will be used in this example. The Serret-Frenet frame formula is obtained by

$\left.T\right|_{t=0}=(1,1,-1)$

$N_{t=0}=\frac{1}{\sqrt{30}}(5,3,-8)$

$\left.B\right|_{t=0}=\frac{1}{\sqrt{30}}(-5,3,2)$.

The curvature and torsion of the curve are $\left.\kappa\right|_{t=0}=\frac{2 \sqrt{30}}{3}$ and $\left.\tau\right|_{t=0}=\frac{7}{30}$, respectively. Consequently, the derivation matrix of SerretFrenet is founded by following matrix

$$
\left(\begin{array}{l}
T^{\prime} \\
N^{\prime} \\
B^{\prime}
\end{array}\right)=\left(\begin{array}{ccc}
0 & 2 \sqrt{30} & 0 \\
2 \sqrt{30} & 0 & 7 / 10 \\
0 & 7 / 10 & 0
\end{array}\right)\left(\begin{array}{l}
T \\
N \\
B
\end{array}\right)
$$

where $v=\left\|\frac{d b^{n}(t)}{d t}\right\|_{L}=3$.

\section{CONCLUSION}

Bezier curves are a type of curve commonly used for ease of use in design geometry. In this paper, we studied on the spacelike Bezier curves with timelike principal normal in Minkowski 3-space. We think that this work will be a guide to research that can be done on this subject in the future.

\section{ACKNOWLEDGEMENT}

The author is grateful to referees for their careful reading of the paper which improved it greatly. 


\section{REFERENCES}

[1]. Ratcliffe J.G., Foundations of hyperbolic manifolds, Graduate texts in mathematics, vol. 149, 2nd edition, 2006.

[2]. Lopez R., Differential geometry of curves and surfaces in Lorentz-Minkowski space. Int. Electron. J. Geom., 7-1 (2014) 44-107.

[3]. Farin G., Curves and Surfaces for ComputerAided Geometric Design, Academic Press, 1996.

[4]. Marsh D., Applied Geometry for Computer Graphics and CAD, Springer Science and Business Media, 2006.

[5]. İncesu M., Gursoy, O., Bezier Eğrilerinde Esas Formlar ve Eğrilikler, XVII Ulusal Matematik Sempozyumu, Bildiriler, Abant İzzet Baysal Üniversitesi, (2004) 146-157.
[6]. Georgiev G.H. Spacelike Bezier Curves in the Three-dimensional Minkowski space, Proceedings of AIP Conference, 1067-1 (2008) .

[7]. Chalmoviansky P., Pokorna B., Quadratic Spacelike Bezier Curves in the three dimensional Minkowski Space, Proceeding of Symposium on Computer Geometry, 20 (2011) 104-110.

[8]. Pokorna B., Chalmovianski P., Planar Cubic Spacelike Bezier Curves in Three Dimensional Minkowski Space, Proceeding of Syposium on Computer Geometry, SCG, 23 (2012).93-98.

[9]. Bükcü B., Karacan M., Bishop Frame of the Spacelike Curve with a Spacelike Principal Normal in Minkowski 3-space, Commun. Fac. Sci. Univ. Ank. Series, A1, 57-1 (2008) 13-22. 